\title{
Layered Bandwidth Management in ATM/SDH Networks
}

\author{
Tai H. Noh \\ Lucent Technologies, Bell Labs Innovations \\ Room 1B-413, Crawfords Corner Rd., Holmdel, NJ 07733 \\ Phone: (908)949-5184, Fax: (908)949-5908, \\ e-mail:tnoh@lucent.com
}

\begin{abstract}
ATM technology offers greater flexibility than STM technology, but in some applications this flexibility has to be compromised to realize a network that performs at least as well as the current STM implementations to achieve guaranteed Quality of Service and favorable economics. This paper presents a layered bandwidth management scheme that exploits the strengths of both STM and ATM technology to realize a more manageable and cost-effective network than is possible by applying only one of these technologies in the integrated ATM and SDH transport network.
\end{abstract}

\section{Keywords}

Bandwidth Management, ATM, STM, SDH, Transport Network Evolution

\section{INTRODUCTION}

The recent trends in ATM/SDH broadband technology have led to high-speed fiber transport links, high-capacity network systems, and multimedia services. These trends have increased the importance of the efficient utilization of bandwidth with guaranteed Quality of Service (QoS) but also the vulnerability to network failures. This paper discusses a bandwidth management strategy for the capacity efficiency in the integrated ATM and SDH transport network. Various end-to-end network protection options are described in the reference [Noh, 1996].

Bandwidth management implies rearranging the current network bandwidth configuration to enhance network adaptability to both expected and unexpected traffic variations. Configuration Management functions in the TMN execute bandwidth management 
control actions, for example, rearranging VP bandwidth when a reconfiguration control parameter, such as VP use, or network traffic exceeds a predefined threshold. A bandwidth reconfiguration interval is based on the traffic behavior in ATM networks. The behavior of an ATM traffic source can be decomposed into four levels: network level, call level, burst level, and cell level [Hui, 1988]. An important attribute of each level is its rate of bandwidth reallocation, which differs substantially from one level to another [Figure 1]. Bandwidth management belongs to the network level. Most of bandwidth reconfiguration schemes proposed to date deal with SDH/PDH networks[Gopal ,1990] [Hasegawa, 1987] and ATM networks [Gerla, 1989] [Monteiro, 1993] [Lee, 1993] [Sato, 1990] separately. There have been very few studies that address overall strategy of bandwidth management to utilize the strengths of both ATM and STM technology.

We divide the network level into two layers- the STM path layer and the ATM VP layer - to introduce the layered bandwidth management concept into the evolving transport network. Sufficient transport bandwidth should be allocated for each level to bound the amount of blocking occurring at the next level.

Layers

Rate of Changes

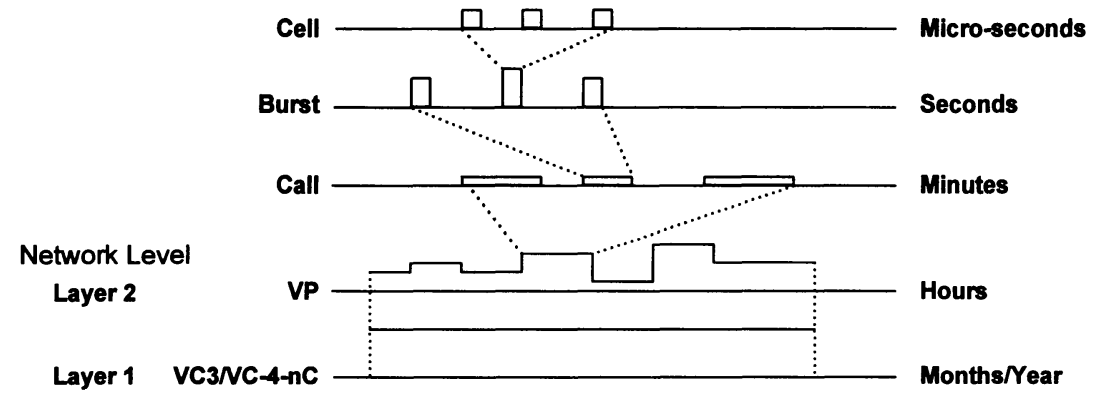

- The facility network should be designed $s 0$ that the probability of failure to set up an VC-4-nC is small.

- An VC-4-nC should be allocated so that the probability of failure to set up VPs is small.

- A VP bandwidth should be allocated so that the probability of failure to use the VP for setting up a call is small.

- A call should be admitted only if the probability of failure due to transporting bursts is small.

- A burst should be transported only if the probability of cell loss is small (The CBR does not have a burst layer).

[Figure 1] Layered Bandwidth Allocation and Rate of Changes

The layered bandwidth management can be supported with either STM cross connection and ATM cross connection as two separate network elements (NE), or via a hybrid STM/ATM cross connection that provides a smooth path in the evolution toward ATM in the transport network. Understanding the technical strength and economics of alternatives will help both network service and system providers develop the most costeffective evolution planning strategy. 
This paper is organized as follows. Section 2 provides transport options for ATM traffic. Section 3 discuses transport network evolution scenarios toward ATM. Section 4 introduces layered bandwidth management. Section 5 provides applications of the layered bandwidth management. Finally, section 6 presents some concluding remarks.

\section{TRANSPORT OPTIONS FOR ATM TRAFFIC}

There are three options to transport ATM traffic over SDH networks. They are embedded ATM transport, hybrid transport, and pure ATM transport within a single SDH tributary.

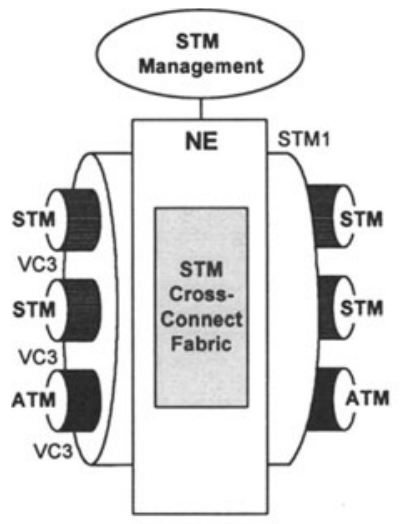

(a) Embedded ATM Transport

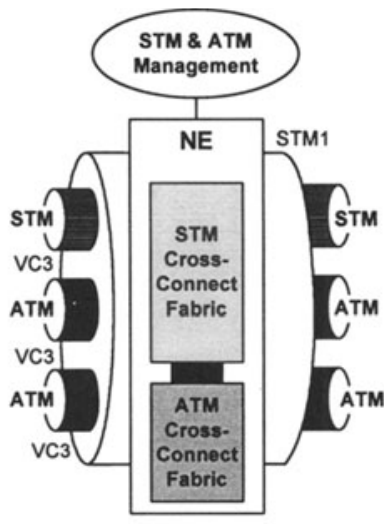

(b) Hybrid ATM/SDH Transport

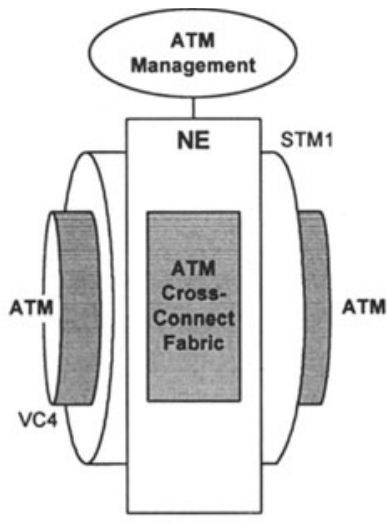

(c) Pure ATM Transport

[Figure 2] Transport Options for ATM Traffic

\section{Embedded ATM transport}

This option corresponds to (a) in Figure 2. ATM cells are mapped to the concatenated mode of SDH payloads by using conventional SDH Network Elements (NE). The SDH NEs provide transport of ATM cells from the customer ATM CPE to the ATM switch and back to the customer ATM CPE. The ATM cells are completely transparent to the SDH NEs. The STM fabric cross-connects VC3 paths under STM management only.

\section{Hybrid Transport}

This option corresponds to (b) in Figure 2. The STM and ATM traffic are combined onto the same SDH pipe over STM tributaries. The NEs have visibility of the ATM cells. The ATM cross-connect fabric in the hybrid NE is used to aggregate ATM traffic to achieve better fills of the concatenated mode of STM tributaries carrying ATM cells. The VC3 paths containing ATM traffic are dropped at the ATM fabric while STM fabric 
provides VC3 cross-connects. Therefore, this NE requires both STM and ATM management.

\section{Pure ATM Transport}

This option corresponds to (c) in Figure 2 and carries all ATM and STM traffic as ATM cells in a single SDH tributary. STM traffic is circuit emulated (if necessary) into ATM cells to maximize the bandwidth utilization of a given SDH pipe by using ATM technologies. The single tributary VC4 in the STM-1 pipe is utilized for ATM traffic under ATM management only.

\section{TRANSPORT NETWORK EVOLUTION TOWARD ATM}

A layered transport network architecture is composed of three layers: the circuit, path, and transmission media layers [ITU-T G.805,1992]. The circuit is an end-to-end connection established/released either dynamically or by short-term provisioning. The transmission media network that interconnects nodes is the physical connection based on long-term provisioning. The path layer bridges these two layers and provides logical connections between terminated node pairs. This path layer plays an important role in constructing flexible ATM/SDH networks.

\section{(a) Embedded ATM}

(b) STM/ATM Hybrid

(c) $100 \%$ ATM Traffic

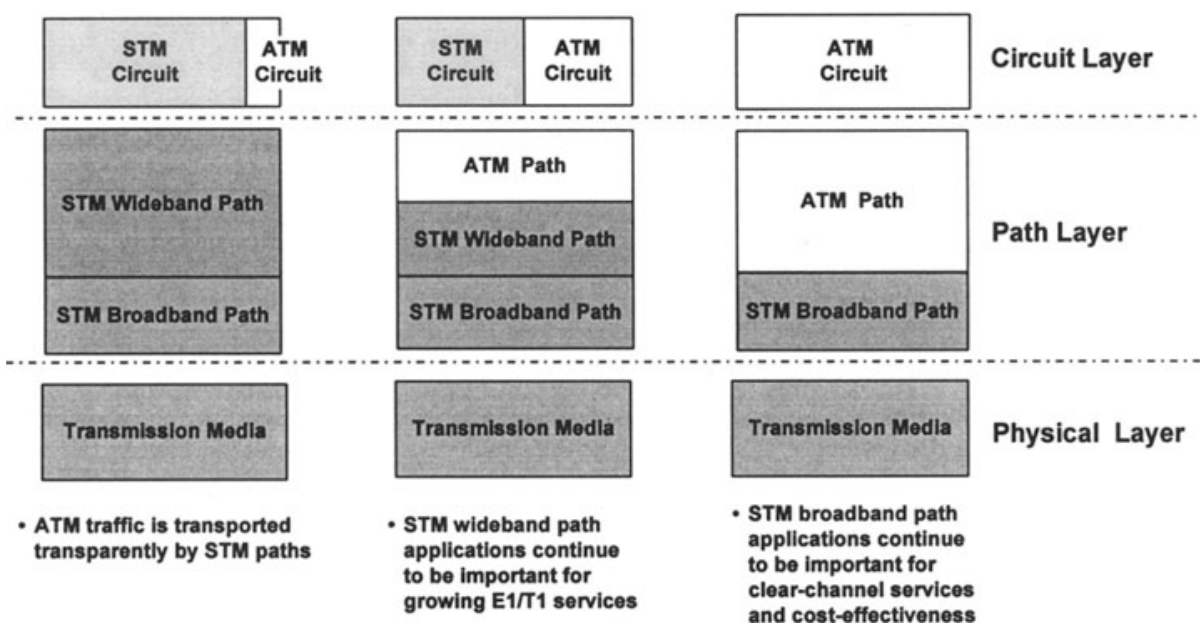

[Figure 3] Transport Network Evolution toward ATM

The path layer is further divided into three different paths: STM wideband, STM broadband, and ATM VP in the evolving transport network [Figure 3]. The STM wideband and broadband path layers have been constructed in the existing SDH networks by using SDH cross connect systems or Add/Drop Multiplexers (ADM). The Virtual Path (VP) extends the concept of path layers to ATM networks. To enhance network 
reconfiguration capability, selecting the right network element to support the three different paths is one of the key concerns in the evolving network from the perspective of guaranteeing QoS and favorable economics. Thus, it is very important to examine both circuit and path layers in the network evolution scenarios to minimize the cost and QoS degradation associated with the circuit emulation between STM and ATM networks.

In the embedded ATM network [(a) in Figure 3], SDH paths provide transparent transport of ATM traffic. Switching of ATM traffic is performed by ATM edge switches located in the access or CPE network and ATM hub switching systems in the junction or interoffice network. An ATM Service Access Multiplexer (SAM) can be used at the edge of the public ATM network to provide ATM interfaces and adaptations for customer services to help reduce the transport inefficiencies associated with the conventional SDH hierarchical tributary structures.

Service providers may want to add ATM functionality to the SDH NEs for the improvement of network bandwidth and flexible introduction of new services with the inherent benefits of ATM technologies. ATM layer grooming is performed by the ATM path to reduce the number of expensive ATM switching systems [(b) in Figure 3]. STM wideband paths applications continue to be important for the growing E1/T1 services. To utilize the inherent strength of the ATM VP technology, E1/T1 services can be circuit emulated. The E1/T1 traffic would be converted to ATM at the edge, connected as ATM through the network, and converted back to circuits on the other side. There is a potential to reduce the overall network equipment cost because ATM connections may be less expensive than STM wideband connections of the same rate, since there does not need to be tributary processing in different paths.

There are important problems however. First, circuit emulation causes a delay. Second, the circuit emulation cost can not be negligible for large STM traffic. There will be a $13 \%$ bandwidth penalty for circuit emulation (ATM cell overhead). These issues will be more significant in the area where most traffic is added/dropped from/to the local office [Wu, 1994].

As ATM traffic demands increase, the transport option in the transport access network will be changed to pure ATM transport with a single SDH tributary such as STM-1 and STM-4c [(c) in Figure 3]. The ATM traffic in the single SDH tributary will be terminated at the junction network for grooming at the ATM layer. STM broadband path applications play an important role as a container for the ATM traffic.

\section{LAYERED BANDWIDTH MANAGEMENT}

\subsection{Description}

Capacity efficiency can be achieved by two-layered bandwidth management in ATM/SDH networks: the STM path layer and the ATM path layer [Figure 4]. An important attribute of each layer is the operating time scale as described in Figure 1. 
Configuration Management in the TMN executes the ATM path layer management [Layer 2] for the short term traffic variations and STM path layer management [layer 1] for the long term service growth.

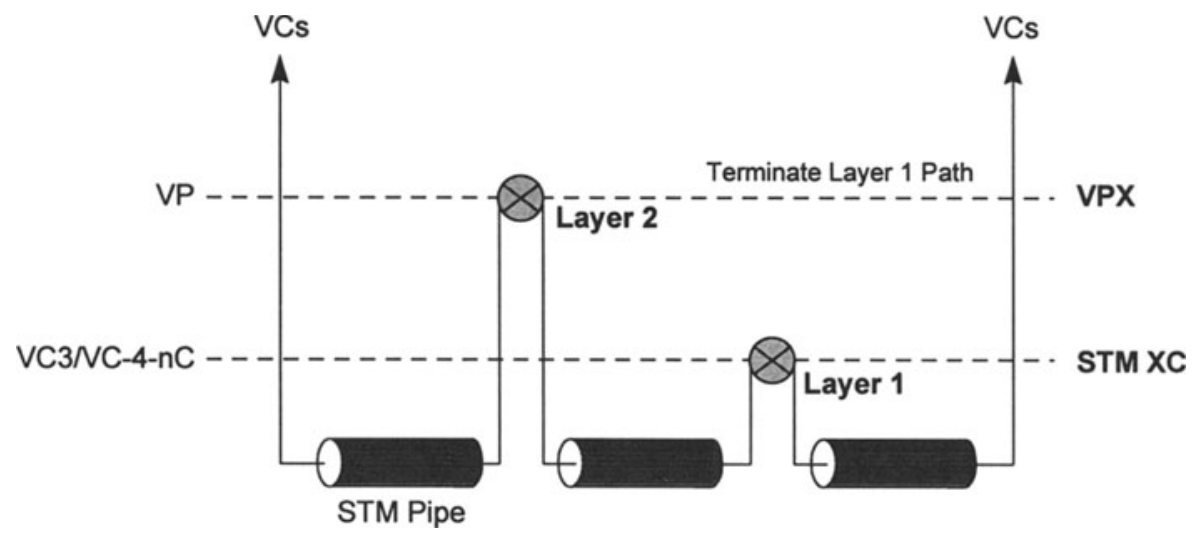

[Figure 4] Layered Bandwidth Management

At the ATM layer, the route and bandwidth of VPs are defined independently. The route is provisioned in the database of the VP terminators and cross-connects. The bandwidth reconfiguration of the VP can be achieved simply by changing datatase values in the VP terminators and cross-connects. This inherent benefit provides more dynamic bandwidth reconfiguration. However, without additional control, cell loss and fluctuation of transmission delay are inevitable due to statistical store and forward transmission. Therefore, the service class that guarantees no cell loss and no intra-network delay jitter is supported by some additional control with extra cost.

In SDH networks, a digital path is established by assigning a time slot for the TDM frame at each cross-connect on the path. Thus, path route establishment and bandwidth assignment are interdependent. Fixed bandwidth digital paths can be established hierarchically. STM cross connect systems are very cost-effective with non-blocking operation [Eng, 1990]. The use of the STM path management between network nodes eliminates the problem of processing cells at gigabit-per-second rates in the high speed backbone network.

The concatenated mode of SDH tributaries such as VC-4-nC provides a container for ATM traffic. For some applications in ATM networks where the bandwidth reallocation unit for traffic demand is VC3/VC-4-nC, switching entire VC3/VC-4-nC ATM containers is more desirable than switching individual ATM cells. The high-speed transport network with multiple ring interconnections is an example in the ATM/STM hybrid network. The interconnected ring networks can be dynamically reconfigured by 
adding or dropping the entire VC3/VC-4-nC tributaries. SDH will be terminated only when grooming at the ATM layer is needed. Characteristics of each layer management is summarized below.

- Layer 2: ATM layer paths -VP

-ATM path traversing ATM VP Cross-Connects (VPXs)

-Bandwidth management for bundle of ATM VCs

-Reduce the number of intermediate ATM switch hops along the path

-Flexible bandwidth allocation

-Efficient bandwidth utilization for service growth and network restoration

- Layer 1: STM layer paths - VC3/VC-4-nC

-STM path traversing ADM/STM Cross-Connects

-Bandwidth management for bundle of VPS

-Reduce the number of intermediate ATM hops along the path

-Reduce buffering delay and nodal processing overhead

-Reduce the number of SDH path termination

\subsection{Examples and Discussions}

To illustrate the operations of each layer management, consider the simple network model that has three ATM Switch (ATM-SW) nodes. A cross-connect system interconnects them for the bandwidth management. The physical links between the cross-connect system and ATM-SWs are STM-1s. The model network accommodates two types of services (class 1 and class 2). No spare capacity is reserved for the network protection. The class 1 is a video service that is coded at $10 \mathrm{Mb} / \mathrm{s}$ and the transport is rate-controlled at $10 \mathrm{Mb} / \mathrm{s}$. The class 2 traffic is a $10 \mathrm{Mb} / \mathrm{s}$ native LAN traffic. Each service class is assigned to different VPs for the segregation of different QoS requirements. The network management system is located at an administrative center that communicates with the ATM-SWs to collect the data of existing demands, from which the required bandwidth for the forecast demands is calculated [Hui, 1988] [Logothetis, 1995].

Figure 5a shows an initial configuration of this model that provides the capability of the layer 1 management. Customer $\mathrm{AB}$ transports three class 1 and three class 2 services between the switch pair $\mathrm{A} \& \mathrm{~B}$. Customer $\mathrm{AC}$ and $\mathrm{BC}$ transport two class 1 and two class 2 services between the switch pair $A \& C, B \& C$ respectively. The customer $A B$ would require two VC3s. Surplus bandwidth on each VC3 is about $8 \mathrm{Mb} / \mathrm{s}$ and $28 \mathrm{Mb} / \mathrm{s}$ respectively since a VC3 provides the customer an available bit rate of $48 \mathrm{Mb} / \mathrm{s}$. The customers $\mathrm{BC}$ and $\mathrm{AC}$ would require a VC3 with $8 \mathrm{Mb} / \mathrm{s}$ surplus bandwidth. 


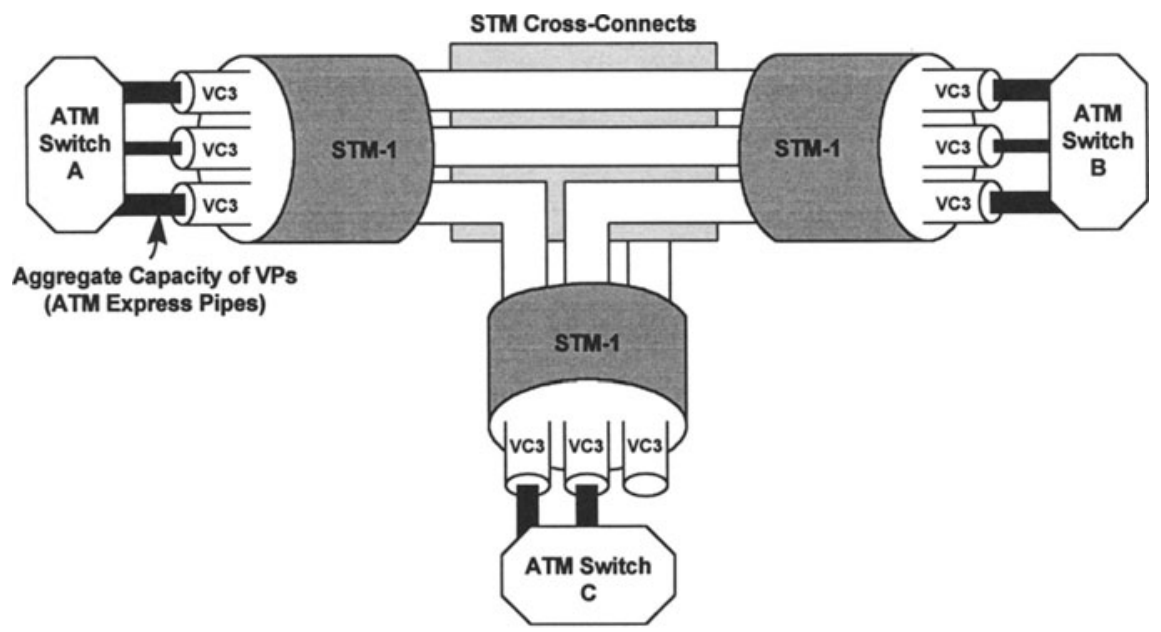

Traffic Demand Decreases Between A \& B

Traffic Demand Increases Between A \& C

Layer 1 Management Leads to Inefficient Bandwidth Utilization

[Figure 5a]: Layer 1 management

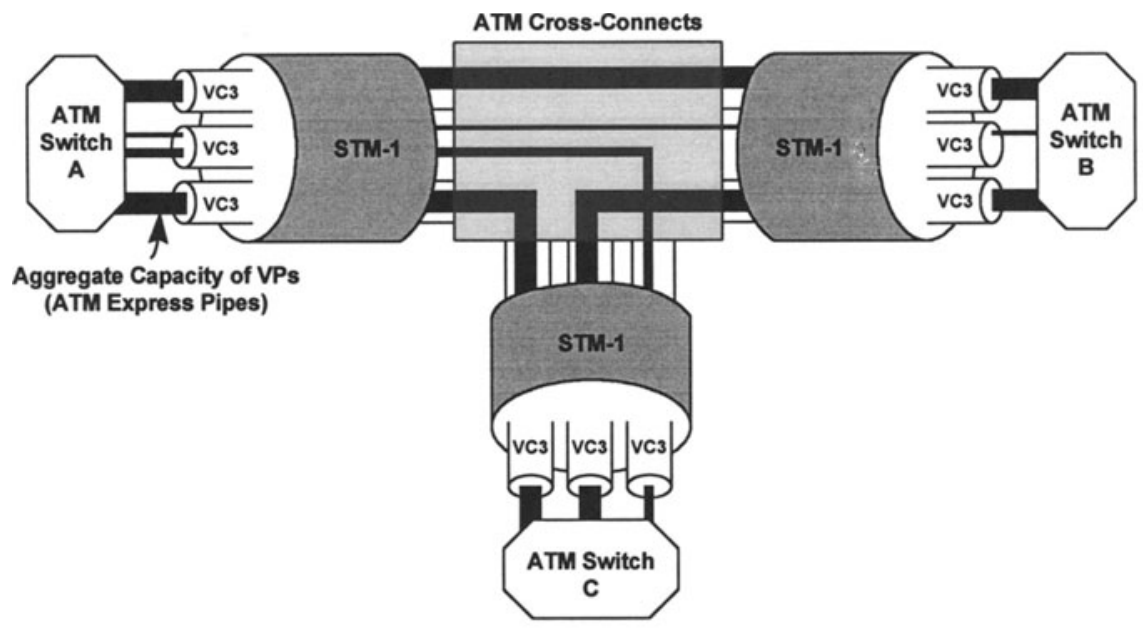

Traffic Demand Decreases Between A \& B

Traffic Demand Increases Between A \& C

Layer 2 Management Provides Efficient Bandwidth Utilization

[Figure 5b]: Layer 2 management 
Now, consider the situation where the forecast traffic demands change. The customer $\mathrm{AB}$ stops subscribing to a class 1 service and the customer $\mathrm{AC}$ wishes to transport two more class 2 services. However, the customer AC can not use the surplus bandwidth on a VC3 between the ATM-SW A and the STM cross connect system that handles VC3 crossconnects. This leads to inefficient bandwidth utilization. This inflexibility issue associated with the boundary of the VC3 tributaries can be resolved by using ATM technology. In Figure 5b, the STM cross-connect system is replaced by an ATM VP cross-connect system for the layer 2 management. Customer AB and AC could now share the same VC3 to transport their ATM traffic.

As ATM traffic demands increase, the transport option in the transport access network will be changed to pure ATM transport with a single SDH path as described in the section 2 and section 3 . Figure $5 \mathrm{c}$ shows that bandwidth utilization can be maximized by using a single tributary VC4. There is no barrier to use surplus capacity.

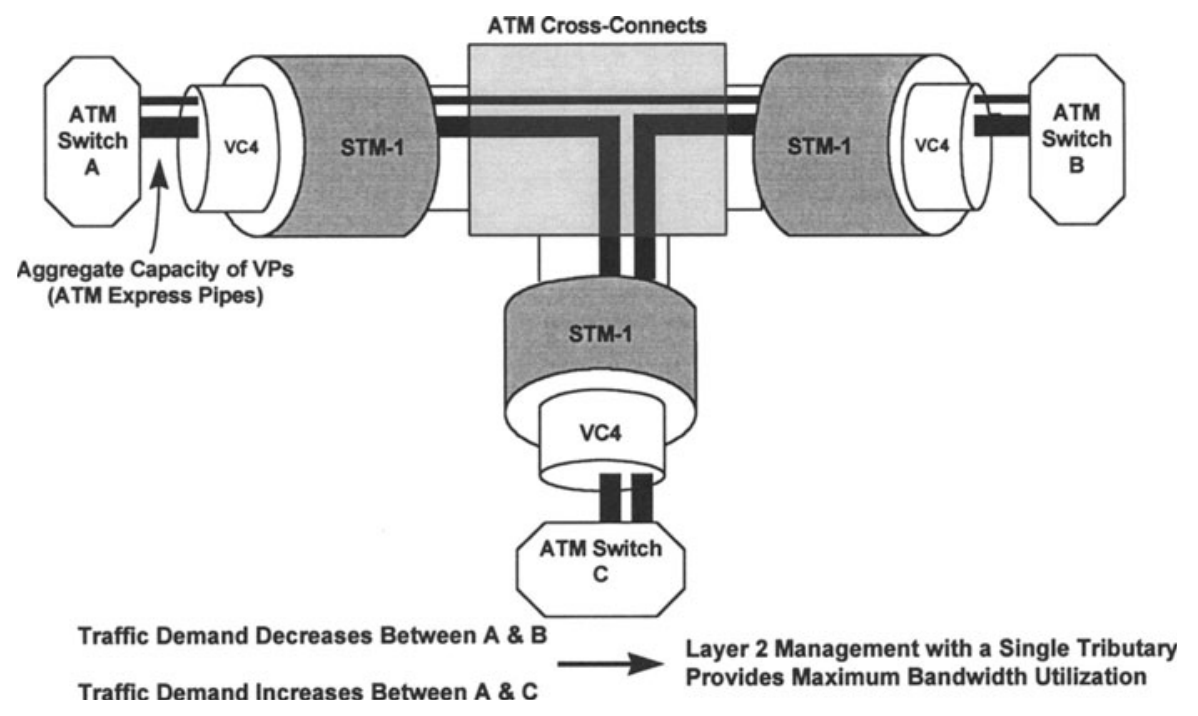

[Figure 5c]: Layer 2 management with a single SDH tributary

The discussion of this example leads to the following conclusions:

- Layer 2 Bandwidth Management is more flexible and bandwidth efficiency can be maximized with the single STM tributary for ATM traffic.

- Layer 1 Bandwidth Management leads to inefficient bandwidth utilization due to the hierarchical structure of the STM tributaries. However, this is no longer an issue if the individual STM express pipe already has significant traffic aggregation. 


\section{NETWORK APPLICATIONS}

The ATM transport network is divided into the transport access network, the transport junction network, and the transport high-speed backbone network. The Telecommunication Management Network (TMN) integrates all operation, administration and maintenance functions for these network components.

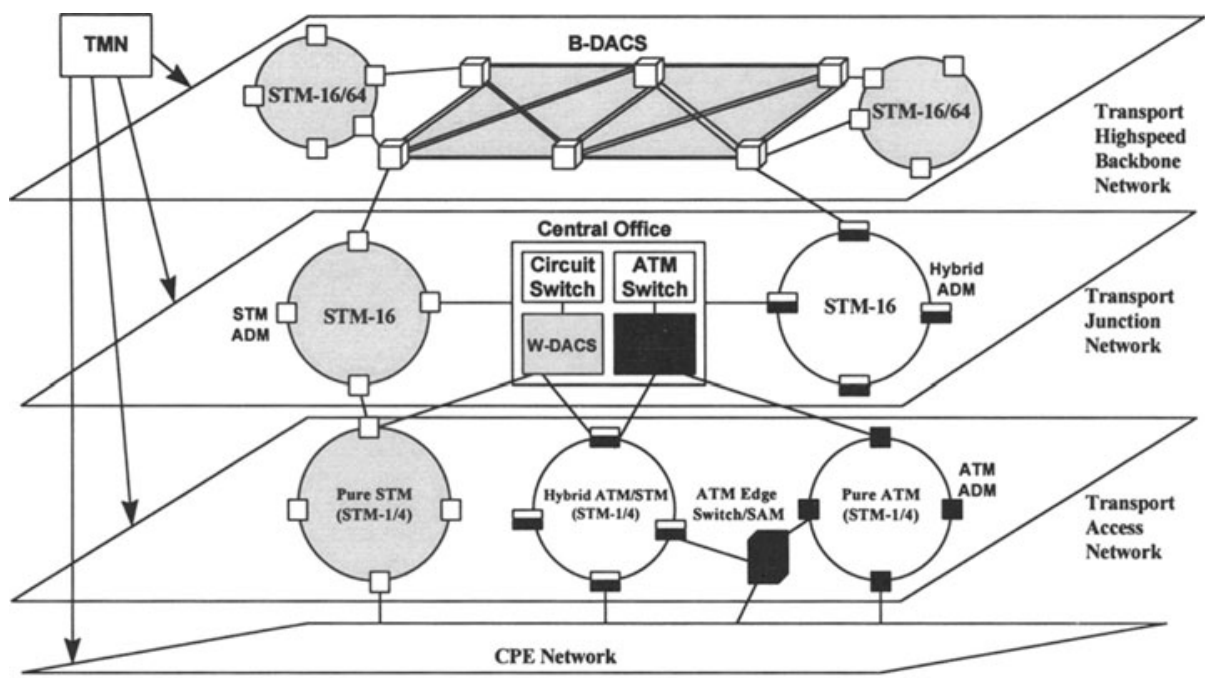

Layer 2 control

Layer 1 control

[Figure 6]: Layered Bandwidth Management at the Transport Network

The transport access network collects traffic from a set of end-user CPE network elements and multiplexes it to one or more designated nodes in the network. SDH/ATM rings connect businesses to the network and can be used for residential access as well. For ATM services the transport access network provides bandwidth management dynamically by using SAM and /or ATM edge switches to best utilize the bandwidth [Layer 2 management].

The junction transport network provides grooming, consolidation, and segregation of different facilities and services in the transmission network between switching systems and between carrier terminals. Grooming is a function that allows use of both incoming and outgoing facilities by the cross-connections of tributaries. Various types of DACSs have been deployed for these network requirements. The importance of this grooming function continues to increase as networks evolve to ATM. Figure 6 shows ATM VPX for the layer 2 management and wideband DACS (W-DACS) for the STM wideband service grooming. The role of W-DACS continues to be important for the growing E1/T1 services as described earlier in the section 3. The ATM/STM hybrid ADMs can be 
effectively utilized to accommodate the transport access network traffic in the high-speed optical transmission lines.

The transport backbone network uses very high capacity fibers that are administered at the broadband granularity [Layer 1 management]. STM based high speed STM-16/64 rings and broadband SDH cross connects are used in this network to provide STM express pipes that are filled by the Layer 2 management at the access and junction networks.

Applicable Areas of each layer of management in the ATM/SDH transport networks are summarized as follows:

Layer 2 Bandwidth Management is appropriate for dynamic bandwidth reallocation

- Edge Network

- ATM Service Access Multiplexer (SAM) and/or ATM Edge Switch

- ATM/STM hybrid rings for low ATM traffic demand and pure ATM rings for high ATM traffic demand

- Junction Network

- ATM mesh network (ATM VPX) and ATM/STM hybrid rings

Layer 1 Bandwidth Management is appropriate for quasi-static bandwidth reallocation

- SDH rings and ATM/STM rings for ring interconnections between transport backbone rings(STM-16/STM-64) and transport access rings (STM-1/STM-4)

- Broadband STM mesh network (B-DACS)

\section{CONCLUDING REMARKS}

We have discussed a bandwidth management strategy in ATM/SDH networks using the strengths of both STM and ATM technology. We have proposed the applicable areas of each layer of bandwidth management from the perspective of long-term transport network evolution toward ATM.

The recommendations are summarized as follows:

- Current STM cross-connect applications continue to be important.

- Layer 1 control is appropriate for quasi-static bandwidth reallocation and applicable to high speed backbone network. 
- Layer 2 control is appropriate for dynamic bandwidth reallocation and applicable to the grooming at edge and junction network.

- Layer 2 management may replace wideband STM path cross-connects if stringent cost targets can be met and operations issues addressed for applications not sensitive to delay.

- Terminate STM path only when the ATM processing is needed. If the entire network is controlled by the layer 2 management, the SDH path will be terminated at every node even if ATM processing is not needed.

\section{ACKNOWLEDGEMENT}

The author would like to thank Stuart Waldman and Dennis Doherty for their reviews and comments. A number of others provided useful comments on the earlier drafts, including Bill Goers, Jack Davis, Fred Feldman, Mark Wilson, Patrice Lamy and Kevin Sparks.

\section{REFERENCES}

K.Y. Eng, R.D. Gitlin and M.J. Karol (1990) A Framework For A National Broadband (ATM/B-ISDN) Network," IEEE Globecom'90, pp. 308.7.1-308.7.6

M. Gerla, S. Monteiro, and R. Pazos (1989) Topology Design and Bandwidth Allocation in ATM Networks," IEEE JSAC, SAC-7(8), pp. 1253-1262

G. Gopal, C. Kim, and A. Weinrib (1990) Dynamic Network Configuration Management," ICC'90, Vol. 2, pp. 302.2.1-7

S. Hasegawa, A. Kanemasa, H. Sakaguchi, and R. Maruta (1987) Dynamic reconfiguration of Digital Cross-Connect Systems with network control and management," Globecom'87, pp. 1096-1100

J. Y. Hui (1988) Resource Allocation for Broadband Networks, IEEE J. On Select. Area in Communication, Vol.6, No.9, pp. 1598-1608.

ITU-T G.805 (1992) Architectures of Transport Networks Based on the Synchronous Digital Hierarchy (SDH)

M. Lee and J. Yee (1993) A Design Algorithm for Reconfigurable ATM Networks," IEEE Infocom, pp. 2a.1.1-2a.1.8 
M. Logothetis, Michael and S. Shioda (1995) Medium-Term Centralized Virtual-Path Bandwidth Control Based on Traffic Measurements," IEEE Transactions on Communications, Vol. 43, No. 10, October 1995

S. Monteiro and M. Gerla (1993) Topological Reconfiguration in ATM Networks," IEEE Inforcom, pp. 267-271

T. Noh (1996) End-to-end Self-healing ATM/SDH Networks," IEEE Globecom'96, pp. 1877-1881

K. Sato and I. Tokizawa (1992) Flexible Asynchronous Transfer Mode Networks Utilizing Virtual Paths," ICC'90, pp. 318.4.1-318.4.8

T. Wu, J. Bartone, and V. Kaminisky (1994) A Feasibility Study of ATM Virtual Path Cross-Connect Systems in LATA Transport Networks," IEEE Globecom'94, pp. 14211427

\section{BIOGRAPHY}

Tai H. Noh is a Member of Technical Staff at Bell Laboratories/Lucent Technologies, where he is currently involved in the SDH/ATM integration, end-to-end network and network systems evolution planning, and end-to-end self-healing SDH/ATM network architecture. He joined Bell Laboratories in 1987. He holds a B.S. from Seoul National University, an M.S. from Rensselaer Polytechnic Institute, and a Ph.D. in Computer Information Engineering from Stevens Institute of Technology. 